\title{
The functional gastrointestinal disorders and the Rome II process
}

Man should strive to have his intestines relaxed all the days of his life.

Moses Maimonides, AD 1135-1204

$A$ good set of bowels is worth more to a man than any quantity of brains.

Josh Billings (Henry Wheeler Shaw), AD 1818-1885

\section{Toward a new understanding of the functional} gastrointestinal disorders

For centuries, physicians and historians have recognized that it is common for maladies to afflict the intestinal tract, producing symptoms of pain, nausea, vomiting, bloating, diarrhea, constipation, difficult passage of food or feces, or any combination. ${ }^{1}$ When these symptoms are experienced as severe, or when they impact on daily life, those afflicted often attribute the symptoms to an illness and seek medical care. Traditionally, the physicians caring for these patients will search for inflammatory, infectious, neoplastic, and other structural abnormalities to make a specific diagnosis and offer specific treatment. Yet as has been common in medical practice, ${ }^{2}$ when no structural etiology is found, the patient is diagnosed as having "functional" symptoms and is treated symptomatically.

Until recently, the limited scientific knowledge about the pathophysiology of these symptoms, and the need to diagnose by excluding "organic" disease, has led physicians to feel uncertain about the legitimacy of these symptoms as bona fide disorders. ${ }^{3}$ Some have felt insecure in their ability to manage patients with these conditions, and might even avoid caring for patients with these complaints. But over the past two decades, two important processes have occurred to legitimize these conditions, and to increase attention toward the research and clinical care of patients with functional gastrointestinal disorders (FGID). The first has been a shift in conceptualizing these disorders from a disease-based, reductionistic model, where the effort is directed toward identifying a single underlying biological etiology, to a more integrated, biopsychosocial model of illness. ${ }^{45}$ The latter model allows for symptoms to be understood as physiologically multidetermined (e.g., altered motility, enhanced visceral sensitivity, brain-gut dysregulation) and as modifiable by sociocultural and psychosocial influences. The second change has been the remarkable growth in investigative techniques that support this newer conceptualization of brain-gut interactions: improved motility assessment, the development of the barostat, imaging of the brain (positron emission tomography (PET), functional magnetic resonance imaging (fMRI)), standardized psychological diagnostic instruments, and the molecular investigation of brain-gut peptides. As a result, over the past two decades, there has been a 10 -fold increment in Medline citations about irritable bowel syndrome (IBS), a much larger number of symposia and presentations at national and international meetings about the FGIDs, and increased effort by pharmaceutical companies to identify and test receptoractive agents to treat these disorders.

These developments built support for an international effort to characterize and classify the FGIDs by investigators and clinicians, pharmaceutical regulatory organizations, pharmaceutical companies, and federal research agencies. The Multinational Working Teams to Develop
Diagnostic Criteria for Functional Gastrointestinal Disorders (Rome Committees) began in the mid 1980s as a series of committees that developed consensus criteria for over 20 FGIDs and published them in several documents in Gastroenterology International. These documents were eventually updated and compiled into a book ${ }^{6}$ as the Rome criteria.

In this supplement, we present the second major effort of the Rome committees: the update of our growing knowledge of the FGIDs over the past five years. We include five articles that detail the FGIDs based on anatomic region, (esophageal, gastroduodenal, bowel, biliary, anorectal), and a separate article on pediatric FGIDs. We also present several articles summarizing current knowledge on the physiologic (motility/sensation), basic science (brain-gut), and psychosocial aspects of the FGIDs, and provide recommendations for the design of treatment trials for these disorders. In addition, we include the revised (Rome II) diagnostic criteria for the FGIDs developed by consensus of experts and based on existing evidence. Finally, as we enter the new millennium, we offer recommendations for future studies that will help advance this growing field.

In this introductory article, I will review the rationale and limitations of a symptom-based diagnostic (Rome II) classification system, discuss the process through which the criteria and consensus information were developed, and briefly summarize the pathophysiological knowledge that is evolving about the FGIDs. More detailed information is provided in the other articles written by the 10 committees.

\section{Rationale for symptom-based diagnostic criteria}

The Rome II classification system is based on the premise that for each disorder there are symptom clusters that "breed true" across clinical and population groups. While not completely true (see Qualifications, below), this presumption provides a framework for identification of patients for research, which can be modified as new scientific data emerge. The rationale for classifying the FGIDs into symptom-based subgroups has three bases. ${ }^{7}$

\section{SITE-SPECIFIC DIFFERENCES}

Patients with FGIDs report a wide variety of symptoms affecting different regions of the gastrointestinal tract. These symptoms have in common disturbances in sensory and/or motor gastrointestinal function, which may overlap across anatomic regions. However, epidemiological studies using factor analysis and other methods provide evidence for the existence of site-specific syndromes. ${ }^{8}{ }^{9}$ The clinical value of separating the functional gastrointestinal symptoms into discrete conditions (table 1) is that they can be reliably diagnosed and more specifically treated. The FGIDs are classified by anatomic region: esophageal (A), gastroduodenal (B), bowel (C) and functional abdominal pain (D), biliary (E), and anorectal (F). Within each anatomic category site, there can be several disorders, each with specific clinical features. For example, the functional bowel disorders (C), which include IBS (C1), functional abdominal bloating (C2), functional constipation (C3), and functional diarrhea (C4), are all functional bowel disorders attributed to the colon and rectum. Yet IBS, having pain associated with change in bowel habit, is distinct from functional diarrhea, which is characterized by loose stools 
Table 1 Functional gastrointestinal disorders

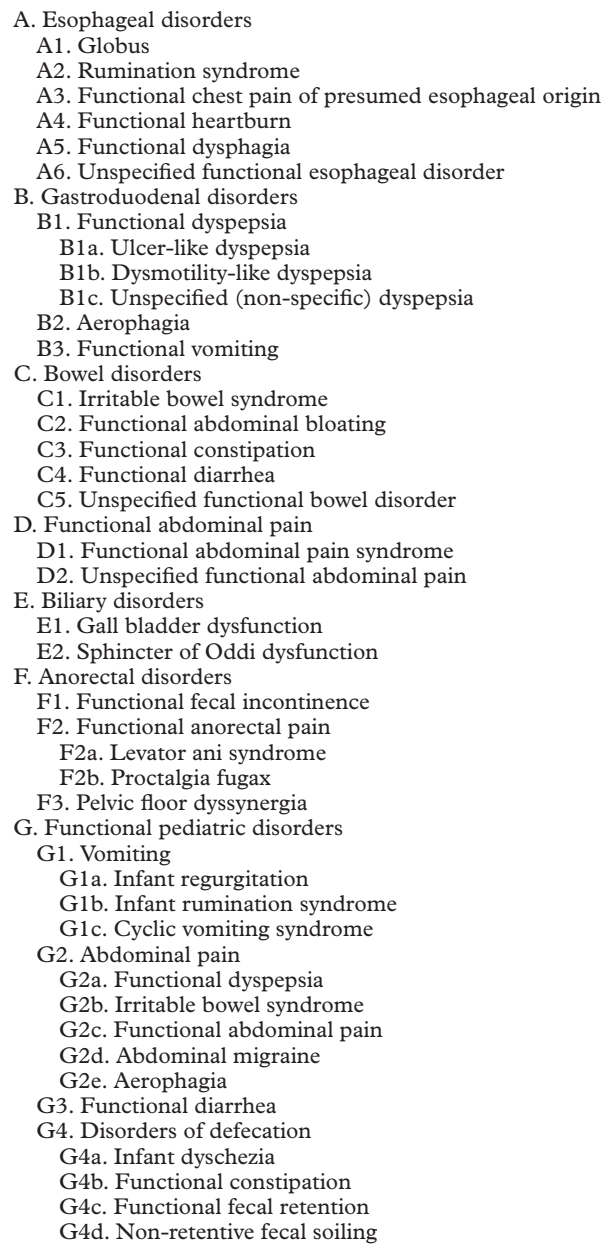

and no pain. Presumably, each condition will have different diagnostic and treatment approaches.

\section{EPIDEMIOLOGICAL DATA}

Epidemiological studies show similar prevalences for these conditions across various studies and populations. The frequencies of bowel symptom patterns reported from England, France, and China are nearly identical (see Functional bowel disorders and Functional abdominal pain). In addition, a factor analysis study using two community samples ${ }^{10}$ identified an irritable bowel factor, and these symptoms were very similar to those developed from a clinical population of patients with IBS using discriminant function analysis (Manning criteria). ${ }^{11}$

\section{NEED FOR DIAGNOSTIC STANDARDS OF CLINICAL CARE AND RESEARCH}

As, by definition, there are no physiological features of diagnostic value, and because it is symptoms that patients bring to physicians, the use of a symptom-based classification system is rational. Symptom-based criteria, used in psychiatry (e.g., DSM-IV) ${ }^{12}$ and rheumatology ${ }^{13}$ and most recently the Rome I criteria, ${ }^{6}$ are becoming increasingly accepted within gastroenterology. Symptom-based criteria help guide the diagnostic and treatment approach, reduce the ordering of unneeded diagnostic tests, and help to standardize the selection of patients for clinical trials.

QUALIFICATIONS FOR THE USE OF SYMPTOM-BASED CRITERIA Certain limitations and qualifications need to be considered. ${ }^{7}$
Other diseases may coexist that need to be excluded

The high frequency of the FGIDs assures their coexistence with other (organic) diseases. In fact, IBS and inflammatory bowel disease (IBD) seem to have a greater than chance association, ${ }^{14}$ and IBD may even predispose to IBS. ${ }^{15}$ Similarly, Helicobacter pylori needs to be excluded and/or treated among patients with functional dyspepsia. So, it is necessary to exclude other diseases before a functional gastrointestinal designation can be applied.

\section{Symptoms may overlap with other functional gastrointestinal disorders}

Because different FGIDs may coexist, the criteria need to allow for hierarchical classification of the FGIDs. For example, if criteria for both IBS (C1) and functional dyspepsia (B1) are fulfilled, then both diagnoses co-exist. However, if criteria for IBS are fulfilled, but the patient also has upper gastrointestinal (dyspeptic-like) pain that is relieved by defecation, then only IBS exists. Also, an unspecified designation (e.g., unspecified functional bowel disorder) permits the inclusion of patients with functional gastrointestinal symptoms who do not meet more specific categories.

Symptoms must be present for at least 12 weeks over the previous year

To make a diagnosis of a FGID, symptoms should be present for at least 12 weeks out of the previous year. There are certain exceptions-for example, chronic functional abdominal pain requires six months of symptoms, and certain pediatric and anorectal disorders require only several weeks of symptoms. The 12-week qualification is a change from the Rome I criteria, which only required that symptoms be present in the previous three months. The reasons for this change are that FGIDs are conditions that have a waxing and waning course, and (particularly for epidemiological surveys) symptoms might not have been present in the previous three months, but may have existed prior to that time. The 12 weeks need not be consecutive, and within each week, symptoms are only required for $1 / 7$ days.

In addition, we recognize the difficulty in translating the time requirement into a questionnaire. For some purposes, investigators may choose the last three months as the time requirement. This choice may be preferred for entry into clinical trials.

Diagnostic categories do not include psychosocial criteria Although psychosocial disturbances can affect the onset, course and outcome of the FGIDs ${ }^{16}$ (see Psychosocial aspects of functional gastrointestinal disorders), they are not required for diagnosis, as they do not occur in people with FGID who do not seek health care. ${ }^{17} 18$

\section{Evidence is determined by clinical consensus and existing} evidence

The proposed diagnostic criteria are derived from the consensus of experts in the field (the committees and their consultants). Using the Rome I consensus criteria as a starting point, the committees had to justify all changes for the Rome II criteria based on existing scientific data, or on a rational recommendation agreed upon by the committee, the Coordinating Committee, and the reviewers. Also, these new criteria will be tested in future studies now underway, and this will form the basis for future modifications of the criteria.

The process of developing diagnostic criteria

The process for developing these criteria is a rigorous one. The consensus process was initiated by Professor Aldo 
Torsoli for the International Congress of Gastroenterology in Rome (Roma '88). Professor Torsoli charged the committees $^{19}$ to use a Delphi method ${ }^{20}$ of decision-making, which fosters a team effort to produce consistency in opinion, or consensus (although not necessarily total agreement) for difficult questions not easily tackled. In 1995, the Rome II Coordinating Committee took on the responsibility of carrying these activities further using a rigorous four year, 10-step process:

(1) The Coordinating Committee identified individuals with international research and clinical expertise to chair each of the 10 committees. Each Chair was charged to develop a manuscript for this supplement and an expanded manuscript for an upcoming book.

(2) Each Chair, in consultation with the Coordinating Committee, identified a Co-Chair and recruited an international panel of up to five additional members having similar clinical and investigative skills to create the manuscripts.

(3) Each committee member produced a document in response to specific assignments given by the Chair and Co-Chair. Usually the task was to synthesize the literature regarding the physiological, psychological, diagnostic, and treatment aspects of a particular functional disorder or scientific content area.

(4) Each Chair incorporated all documents into a manuscript that was sent to the entire committee for review.

(5) This process of modification and re-review by the committee was repeated two more times over a two year period.

(6) Each committee met for three days in June 1998 to revise the document, using the Delphi method..$^{20}$ This face-to-face meeting led to consensus on the diagnostic criteria and scientific content.

(7) Each Chair sent the revised document to at least six outside international experts and to scientists in the pharmaceutical industry for review and comment.

(8) The committee Chairs responded to the reviewers' comments either by modifying the manuscripts as requested, or providing a written response that addressed the reviewers' concerns.

(9) The revised manuscripts and the commentaries by the reviewers and authors were then sent to the Coordinating Committee who met in November 1998 and again in February 1999 to review these materials critically, and to submit critiques back to the authors.

(10) Finally, when the document was completed, all members signed off their approval before it was returned back to the Coordinating Committee for a final check on content and style prior to submission for publication.

\section{Observations on the functional gastrointestinal disorders}

In proposing the diagnostic groupings listed in table 1, we recognize that, despite differences in location and symptom features, they share common features with regard to their motor and sensory physiology, central nervous system (CNS) relationships, and the approach to patient care. What follows are general observations and guidelines for these disorders.

\section{ABNORMAL MOTILITY}

It is well recognized that vomiting, diarrhea, acute abdominal pain, incontinence, and many other gastrointestinal symptoms are generated by disturbed gastrointestinal motility. Furthermore, in healthy subjects, strong emotion or environmental stress can lead to increased motility in the esophagus, stomach, small intestine, and colon. The FGIDs are characterized by having an even greater motility response to stressors (psychological or physiological) when compared with responses in normal subjects; candidate electrical activity markers have been proposed which may facilitate these responses. ${ }^{21}$ However, these motor responses are only partially correlated with symptoms and are not sufficient to explain reports of chronic or recurrent abdominal pain.

\section{VISCERAL HYPERSENSITIVITY}

The poor association of pain with gastrointestinal motility in many of the FGIDs (e.g., functional chest pain of presumed esophageal origin (A3), functional dyspepsia (B1), IBS (C1), functional abdominal pain (D)), is explained by more recent studies relating to abnormalities in visceral sensation. ${ }^{22}$ These patients may have a lower pain threshold when tested with balloon distension of the bowel (visceral hyperalgesia), or they may have increased sensitivity even to normal intestinal function (e.g., allodynia), and there may be an increased or unusual area of somatic referral of visceral pain. It is not yet clear whether visceral hypersensitivity in the FGIDs relates to $(a)$ altered receptor sensitivity at the viscus itself, $(b)$ increased excitability of the spinal cord dorsal horn neurons, or (c) altered central modulation of sensation. ${ }^{21}$

Another recent observation is that visceral hypersensitivity may be induced (stimulus hyperalgesia) in response to rectal or colonic distension in normal subjects ${ }^{23}$ and to a greater degree in people with IBS. $^{24}$ So the pain of an FGID may relate to sensitization resulting from chronic motor hyperactivity (e.g., discrete clustered contractions), gastrointestinal infection (e.g., $H$ pylori for functional dyspepsia and enteric infections for IBS (see later) or trauma/ injury to the viscera).

\section{INFLAMMATION}

Certain investigators have proposed that increased inflammation in the enteric mucosa or neural plexi may contribute to symptom development. ${ }^{25}$ This may occur by peripheral sensitization (see earlier), and/or hypermotility activated by induction of mucosal inflammatory cytokines. ${ }^{26}$ This hypothesis follows clinical observations that about one-third of patients with IBS report that their symptoms began after an acute enteric infection, and also, one-third of patients presenting with an acute enteric infection will go on to develop IBS-like symptoms. ${ }^{27}{ }^{28}$

\section{BRAIN-GUT INTERACTIONS}

To bring these observations together, we emphasize a more integrative biopsychosocial understanding of these symptoms as being generated by a combination of intestinal motor, sensory, and CNS activity - the brain-gut axis. ${ }^{29}$ Thus, extrinsic (vision, smell, etc.) or enteroceptive (emotion, thought) information have, by nature of their neural connections from higher centers, the capability to affect gastrointestinal sensation, motility, secretion, and inflammation. Conversely, viscerotopic effects (e.g., nociception) reciprocally affect central pain perception, mood, and behavior. For example, spontaneously induced contractions of the colon in rats lead to activation of the locus coeruleus in the pons, an area closely connected to pain and emotional centers in the brain. ${ }^{30}$ Conversely, increased arousal or anxiety is associated with a decrease in the frequency of migrating motor complex activity of the small bowel. ${ }^{31}$

Brain-gut interactions may also influence postinfectious IBS. In a study of patients admitted for acute gastroenteritis, $23 \%$ developed IBS-like symptoms three months later. When comparing those who developed IBS symptoms with those who did not: (1) psychosocial difficulties (life stress 
and hypochondriasis) predicted who would go on to develop IBS symptoms; (2) altered gut physiology (abnormal motility and visceral hypersensitivity) was present at three months in both groups (i.e., with or without symptoms); and (3) increased inflammatory cells were present only in the group with IBS symptoms. ${ }^{28}$ So although the acute infection led to physiological disturbances in the gut (altered sensation and motility), it seems that the psychological distress contributed to the later experience of the symptoms. The stress may perpetuate IBS symptoms by CNS facilitation of inflammation, via disruption of the hypothalamic-pituitary-adrenal/ cytokine stress pathway. ${ }^{32} 33$

It is now possible to study the brain physiology of patients with FGIDs using PET, fMRI, and related imaging modalities. ${ }^{34}$ These studies may help us to understand the role of the CNS in modulating visceral pain and motility. They may also provide a marker for studying responses to psychological treatments or psychopharmacological agents, as has been shown in analogous studies of psychiatric disorders. It was recently reported that depressed patients with more active anterior cingulate gyrus activity on PET imaging were more likely to respond to antidepressants. ${ }^{35}$

\section{BRAIN-GUT PEPTIDES}

A treatment approach consistent with the concept of braingut dysfunction is likely to involve the neuropeptides and receptors present in the enteric and central nervous systems. Putative agents include primarily 5-hydroxytryptamine and its congeners, the enkephalins and opioid agonists, substance $\mathrm{P}$, calcitonin gene related polypeptide, and cholecystokinin, among others. These neuropeptides have integrated activities on gastrointestinal function and human behavior depending upon their location. Ongoing phase II and III pharmacological treatment trials using agents active at these receptor sites are addressing the diverse, but interconnected symptoms of pain, bowel dysfunction, and psychosocial distress so commonly associated with the FGIDs. The information gained from these studies may increase our understanding of these conditions, as well as possibly provide more effective treatment for patients with these conditions.

\section{PSYCHOSOCIAL FACTORS}

Although psychosocial factors do not define these disorders, and are not required for diagnosis (see later), they are important modulators of the patient's experience and behavior, and ultimately, the clinical outcome. Research on the psychosocial aspects of patients with FGIDs yields three general observations:

- Psychological stress exacerbates gastrointestinal symptoms. Psychological stress or one's emotional responses to stress can affect gastrointestinal function and produce symptoms in healthy subjects, but does so to a greater degree in patients with FGIDs. ${ }^{36}$

- Psychological disturbances modify the experience of illness and illness behaviors such as health care seeking. Although patients with FGIDs show greater psychological disturbance than otherwise healthy subjects and patients with medical disease (see Psychosocial aspects of functional gastrointestinal disorders), the data are drawn from patients seen at referral centers; among non-health care seekers, such disturbances are no different than in the general population. ${ }^{17}{ }^{18}$ So psychosocial factors modulate illness experience and behaviors such as health care seeking. This explains findings associated with psychosocial trauma (e.g., history of sexual or physical abuse) which is found to be more common in referral centers than in primary care, may lower pain
FGID-Conceptual model

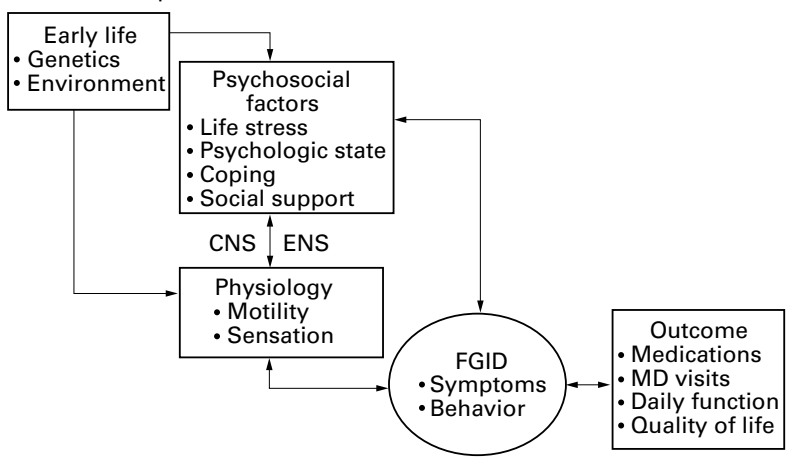

Figure 1 A biopsychosocial conceptualization of the pathogenesis and clinical expression of the functional gastrointestinal disorders (FGID).

CNS, central nervous system; ENS, enteric nervous system.

threshold and symptom reporting, and is associated with a poorer clinical outcome. ${ }^{37}$

- An FGID may have psychosocial consequences. Any chronic illness has psychosocial consequences on one's general well-being, daily function status, one's sense of control over the symptoms, and the implications of the illness in terms of future functioning at work and at home. This is understood in terms of one's healthrelated quality of life (HRQOL). Several new HRQOL instruments have been developed to evaluate patients with FGIDs (see Psychosocial aspects of functional gastrointestinal disorders and Design of treatment trials for the functional gastrointestinal disorders).

\section{A biopsychosocial conceptualization}

As indicated by the findings summarized in the previous section, the links among psychosocial and physiological factors are essential for a full understanding of the mechanisms and varying presentations of the FGIDs. Figure 1 conceptualizes this biopsychosocial understanding of the relationship between psychosocial and physiological factors with functional gastrointestinal symptoms and the clinical outcome. Early in life, genetics and environmental influences (e.g., family attitudes toward bowel training or illness in general, major loss or abuse history, or exposure to an infection) may affect one's psychosocial development (susceptibility to life stress, psychological state, coping skills, development of social support) or the development of gut dysfunction (abnormal motility or visceral hypersensitivity). In addition, the presence and nature of a FGID is determined by the interaction of psychosocial factors and altered physiology via the brain-gut axis. So, one individual afflicted with a bowel disorder, but with no psychosocial disturbances and good coping skills and social support, may not experience the symptoms as distressing enough to seek medical care. Another, having co-existent psychosocial disturbance, high life stress, and poor social support, may experience the symptoms as severe and unmanageable, may see physicians frequently, and have a generally poor outcome.

Based on the observations presented, it is no longer rational to try to discriminate whether physiological or psychologic factors produce pain or other bowel symptoms, dysmotility, or inflammation. Instead, the FGIDs seem to be understood in terms of dysregulation of braingut function, and the task is to determine the degree to which each is operative and remediable.

\section{Concluding comments}

It is with great pleasure and anticipation that we introduce this supplement on the functional gastrointestinal disorders, containing information on over two dozen adult and 
pediatric FGIDs, as well as supplementary information on the basic, physiologic and psychosocial aspects of these conditions. We hope that the information will make a difference in increasing awareness and knowledge relating to the diagnosis and care of patients with FGIDs. This supplement, and the book to follow (Rome II: The Functional Gastrointestinal Disorders published by Degnon Associates, 2000), which will contain more detailed information, are the culmination of four years of effort by over 60 internationally recognized investigators in the field. For further information, contact www.romecriteria.org.

As new scientific data about these disorders accumulate, there will be a need to revise the diagnostic criteria and the information provided relating to the pathophysiology and treatment of these conditions. Therefore, we have set up a process for validation studies and other future activities of the Rome Committees that will permit ongoing review and modification of our current recommendations.

On behalf of the coordinating committee, I would like to thank our sponsors and supporters. From the pharmaceutical industry, the following sponsors merit special commendation: Special sustaining sponsor: Janssen Pharmaceutica; Full sponsors: Astra-Zeneca, GlaxoWellcome, Novartis, Pfizer, Roberts Pharaceuticals, SmithKline Beecham, and Solvay Pharmaceuticals; and Partial sponsors: Procter and Gamble, Sanofi Winthrop, and TAP Holdings. In addition, the following professionals and organizations contributed generously of their time and expertise: the Food and Drug Administration (FDA); the Japan International Gastrointestinal Motility Society; the Functional Brain Gut Research Group; the International Foundation for Functional Gastrointestinal Disorders; the National Institute of Digestive Diseases and Kidney Disorders (NIDDK-NIH); Associazione per la NeUroGastroenterlogia e la Motilità Gastrointestinale (ANEMGI); and the over 53 authors on 10 committees who worked tirelessly to produce these documents. Finally, special thanks go to Ms Carlar Blackman for her creative intelligence and tireless effort to keep the numerous projects and activities underway; to George Degnon for his skills in coordinating our of the meeting in Rome; and Ms Lou Ann Brower for her technical assistance of the meeting in Rome; and Ms Lou Ann Brower for her tech
and genuine interest in producing the best in a final product.

Chair, Coordinating Committee, Multinational Working Teams to Develop Diagnostic Criteria for Functional Gastrointestinal Disorders (Rome II)

D A DROSSMAN

Professor of Medicine and Psychiatry,

Co-Director, UNC Center for Functional GI and Motility Disorders,

Division of Digestive Diseases, 726 Burnett-Womack,

CB\#7080, University of North Carolina at Chapel Hill,

Chapel Hill, NC 27599-7080, USA

1 Drossman DA. Psychosocial and psychophysiologic mechanisms in GI illness. In: Kirsner JB, ed. The growth of gastroenterologic knowledge in the 20th illness. In: Kirsner JB, ed. The growth of gastroenterologic knowled
century. 1st edn. Philadelphia: Lea and Febiger, 1993:419-32.

2 Kroenke K, Mangelsdorff AD. Common symptoms in ambulatory care: incidence, evaluation, therapy, and outcome. Am ₹ Med 1989;86:262-6.

3 Christensen J. Heraclides or the physician. Gastroenterol Int 1990;3:45-8.

4 Engel GL. The need for a new medical model: a challenge for biomedicine. Science 1977;196:129-36.

5 Drossman DA. Presidential address: gastrointestinal illness and biopsychosocial model. Psychosom Med 1998;60:258-67.

6 Drossman DA, Richter JE, Talley NJ, et al (eds). The functional gastrointestinal disorders: diagnosis, pathophysiology and treatment. 1st edn. McLean, VA: Degnon Associates, 1994.

7 Drossman DA. Do the Rome Criteria stand up? In: Goebell H, Holtmann G, Talley NJ, eds. Functional dyspepsia and irritable bowel syndrome: concepts and controversies (Falk Symposium 99). 1st edn. Dordrecht: Kluwer Academic Publishers, 1998:11-18.
8 Whitehead WE. Functional bowel disorders: Are they independent diagnoses? In: Corazziari E, ed. Neurogastroenterology, 1st edn. Berlin: Walter de Gruyter, 1996:65-74.

9 Whitehead WE, Gibbs NA, Li Z, et al. Is functional dyspepsia just a subset of the irritable bowel syndrome? Baillieres Clin Gastroenterol 1998;12:44361

10 Whitehead WE, Crowell MD, Bosmajian L, et al. Existence of irritable bowel syndrome supported by factor analysis of symptoms in two community samples. Gastroenterology 1990;98:336-40.

11 Manning AP, Thompson WG, Heaton KW, et al. Towards positive diagnosis of the irritable bowel. BMF 1978;ii:653-4.

12 American Psychiatric Association. Diagnostic and statistical manual of mental disorders-DSM-IV. 4th edn. Washington, DC: American Psychiatric Association, 1994

13 Schumaker HR, Klippel JH, Robinson DR. Primer on the rheumatic diseases. 9th edn. Atlanta: Arthritis Foundation, 1988

14 Bayless TM. Inflammatory bowel disease and irritable bowel syndrome. Med Clin North Am 1990;49:21-8.

15 Isgar B, Harman M, Kaye MD, et al. Symptoms of irritable bowel syndrome in ulcerative colitis in remission. Gut 1983;24:190-2.

16 Drossman DA, Creed FH, Olden KW, et al. Psychosocial aspects of the functional gastrointestinal disorders. Gut 1999;45(suppl II):II25-30.

17 Drossman DA, McKee DC, Sandler RS, et al. Psychosocial factors in the irritable bowel syndrome: a multivariate study of patients and nonpatients with irritable bowel syndrome. Gastroenterology 1988;95:701-8.

18 Whitehead WE, Bosmajian L, Zonderman AB, et al. Symptoms of psychologic distress associated with irritable bowel syndrome: comparison of community and medical clinic samples. Gastroenterology 1988;95:709-14.

19 Torsoli A, Corazziari E. The WTR's, the Delphic Oracle and the Roman Conclaves. Gastroenterol Int 1991;4:44-5.

20 Milholland AV, Wheeler SG, Heieck JJ. Medical assessment by a delphi group opinion technic. N Engl f Med 1973;298:1272-5.

21 Drossman DA, Whitehead WE, Camilleri M. Irritable bowel syndrome: a technical review for practice guideline development. Gastroenterology 1997; $112 \cdot 2120-37$.

22 Mayer EA, Gebhart GF. Basic and clinical aspects of visceral hyperalgesia. Gastroenterology 1994;107:271-93.

23 Ness TJ, Metcalf AM, Gebhart GF. A psychophysiological study in humans using phasic colonic distension as a noxious visceral stimulus. Pain 1990;43:377-86.

24 Munakata J, Naliboff B, Harraf F, et al. Repetitive sigmoid stimulation induces rectal hyperalgesia in patients with irritable bowel syndrome. Gastroenterology 1997;112:55-63.

25 Collins SM. Is the irritable gut an inflamed gut? Scand f Gastroenterol 1992; 27(suppl 192):102-5.

26 Collins SM. The immunomodulation of enteric neuromuscular function: Implications for motility and inflammatory disorders. Gastroenterology 1996;111:1683-99.

27 McKendrick W, Read NW. Irritable bowel syndrome-post salmonella infection. F Infect 1994;29:1-4.

28 Gwee KA, Leong YL, Graham C, et al. The role of psychological and biological factors in post-infective gut dysfunction. Gut 1999;44:400-6.

29 Mayer EA, Raybould HE. Role of visceral afferent mechanisms in functional bowel disorders. Gastroenterology 1990;99:1688-704.

30 Svensson TH. Peripheral, autonomic regulation of locus coeruleus noradrenergic neurons in brain: putative implications for psychiatry and psychopharmacology. Psychopharmacology 1987;92:1-7.

31 Valori RM, Kumar D, Wingate DL. Effects of different types of stress and or "prokinetic" drugs on the control of the fasting motor complex in humans. Gastroenterology 1986;90:1890-900.

32 Drossman DA. Mind over matter in the postinfective irritable bowel. Gut 1999;44:306-7.

33 Drossman DA. Psychosocial factors in ulcerative colitis and Crohn's disease. In: Kirsner JB, ed. Inflammatory bowel disease. 5 th edn. Philadelphia: WB Saunders, 1999 (in press).

34 Aziz Q, Thompson DG. Brain-gut axis in health and disease. Gastroenterology 1998;114:559-78.

35 Mayberg HS, Brannan SK, Mahurin RK, et al. Cingulate function in depression: a potential predictor of treatment response. Neuroreport 1997;8:1057-61.

36 Whitehead WE, Crowell MD, Robinson JC, et al. Effects of stressful life events on bowel symptoms: subjects with irritable bowel syndrome compared to subjects without bowel dysfunction. Gut 1992;33:825-30.

37 Drossman DA, Talley NJ, Olden KW, et al. Sexual and physical abuse and gastrointestinal illness: review and recommendations. Ann Intern Med 1995;123:782-94.

visit our website at:

www.romecriteria.org 